\title{
Linear assemblies of magnetic nanoparticles as MRI contrast agents
}

Serena A. Corr, Stephen J. Byrne, Renata Tekourite, Carla J. Meledandri, Dermot

F. Brougham, Marina Lynch, Christian Kerskens, Laurence O' Dwyer and

Yurii K. Gun'ko

\section{Supporting Information}

Index

1. Experimental details

S1

2. TEM images of PSSS-Mag nanocomposites S4

3. IR spectra of PSSS-Mag nanocomposites S6

4. Raman spectra of PSSS-Mag nanocomposites S7

5. XRD patterns of PSSS-Mag nanocomposites S8

6. Field dependent longitudinal and transverse relaxation data for magnetic fluid PSSS-Mag1 S9

7. MR images of rat administered with PSSS-Mag1 S10

8. MR videos of rat administered with PSSS-Mag1 S11

\section{Experimental section}

\section{a. Materials}

Ferric chloride hexahydrate $\left(\mathrm{FeCl}_{3} \cdot 6 \mathrm{H}_{2} \mathrm{O}\right)$ and ammonium hydroxide $\left(\mathrm{NH}_{4} \mathrm{OH}, 0.88 \mathrm{M}\right)$ were obtained from BDH Chemicals. Ferrous chloride tetrahydrate $\left(\mathrm{FeCl}_{2} \cdot 4 \mathrm{H}_{2} \mathrm{O}\right)$ and poly(sodium 4-styrenesulfonate) (average molecular weight 70,000 $\mathrm{g} / \mathrm{mol}$ ) were obtained from Aldrich. Millipore water was deoxygenated by boiling for 30 minutes and drying under argon. FTIR spectra $\left(200-4000 \mathrm{~cm}^{-1}\right)$ were recorded by diffuse reflectance using a Perkin Elmer Spectrum One FT-IR spectrophotometer. Samples were pressed into $\mathrm{KBr}$ disks and spectra were recorded. Raman spectra were measured with a Renishaw 1000 micro-Raman system. The excitation wavelength was $514.5 \mathrm{~nm}$ from an $\mathrm{Ar}^{+}$ion laser (Laser Physics Reliant 150 Select Multi-Line) with a typical laser power of $3 \mathrm{~mW}$ in order to avoid excessive heating. The $100 \times$ magnifying objective of the Leica microscope was capable of focusing the beam into a spot of approximately $1 \mu \mathrm{m}$ diameter. X-Ray powder diffraction was performed 
using a Siemens-500 X-Ray diffractometer. Powder samples were deposited on silica glass using silica gel to adhere the sample to the glass surface. Overnight spectra were run for all samples. Diffractograms were then compared to the JCPDS database. Transmission electron microscopy (TEM) images were taken on a Hitachi H-7000. The TEM was operated at a beam voltage of $100 \mathrm{kV}$. Samples for TEM were prepared by deposition and drying of a drop of the powder dispersed in ethanol or distilled water onto a formvar coated 400 mesh copper grid. ${ }^{1} \mathrm{H} \mathrm{T}_{1}$ measurements (NMRD) were made in the frequency range $10 \mathrm{kHz}-20 \mathrm{MHz}$ using a Spinmaster Fast Field Cycling Relaxometer operating at a measurement frequency of 9.25 MHz.

\section{Preparation of PSSS-Mag nanocomposites}

$\mathrm{FeCl}_{3} .6 \mathrm{H}_{2} \mathrm{O}(1.1 \mathrm{~g} ; 4 \mathrm{mmol})$ and $\mathrm{FeCl}_{2} .4 \mathrm{H}_{2} \mathrm{O}(0.4 \mathrm{~g} ; 2 \mathrm{mmol})$ were dissolved in $100 \mathrm{~mL}$ deoxygenated water. Solutions of polyelectrolyte were prepared in $10 \mathrm{~mL}$ volumes. Polyelectrolyte solutions were added to predetermined volumes of iron solutions as shown in Table 1.

Table 1: Experimental setup for the preparation of PSSS-Mag nanocomposites

\begin{tabular}{|c|c|c|c|c|}
\hline $\begin{array}{c}\text { Sample } \\
\text { name }\end{array}$ & $\begin{array}{c}\text { Volume of } \\
\text { iron solution } \\
(\mathbf{m L})\end{array}$ & $\begin{array}{c}\text { Polyelectrolyte } \\
\text { concentration (M) }\end{array}$ & $\begin{array}{c}\text { Fe:PSSS } \\
\text { ratio }\end{array}$ & $\begin{array}{c}\text { Fe:monomer } \\
\text { ratio }\end{array}$ \\
\hline PSSS-Mag1 & 10 & $3.6 \times 10^{-4} \mathrm{M}$ & 168 & $1 / 2$ \\
\hline PSSS-Mag2 & 5 & $3.85 \times 10^{-5} \mathrm{M}$ & 1050 & 3 \\
\hline PSSS-Mag3 & 2.5 & $1.4 \times 10^{-5} \mathrm{M}$ & 2100 & 6 \\
\hline
\end{tabular}

The solutions were allowed to stir at room temperature for 15 minutes. Magnetic nanoparticles were precipitated via the addition of ammonia solution until the $\mathrm{pH}$ reached 9. The resulting black precipitates were washed with Millipore water $(5 \times 20 \mathrm{~mL})$ and the final washes used in MRI experiments and TEM analysis. The precipitate was dried under vacuum and analysed by IR and Raman spectroscopy and XRD patterns. 


\section{MRI Materials and Methods}

Animals were imaged using a Bruker 7T Biospec Scanner (Bruker Medizintechnik, Karlsruhe, Germany). The following image sequences were obtained: (a) Echo Planar Imaging (EPI). Repetition Time (TR) = $700 \mathrm{~ms}$; Echo Time (TE) $=15$ ms. Flip angle $=90$ degrees. (b) T1-weighted Fast Low Angle Shot (FLASH). TR = $8.425 \mathrm{~ms} ; \mathrm{TE}=2.96$. Flip angle $=30$ degrees. For EPI, 1 coronal slice was acquired with $1 \mathrm{~mm}$ thickness every $0.7 \mathrm{sec}$. There were 50 repetitions and total scan time was $35 \mathrm{sec}$. The EPI factor $=46$; matrix $=64 \times 64$; field of view $(F O V)=3 \mathrm{~cm} \times 3 \mathrm{~cm}$. For FLASH, 1 coronal slice was acquired with $1.5 \mathrm{~mm}$ thickness every $0.8 \mathrm{sec}$. There were 60 repetitions and total scan time was $48.529 \mathrm{sec}$. Matrix $=128 \times 128 ;$ FOV $=3$ $\mathrm{cm} \times 3 \mathrm{~cm}$. For both the FLASH and EPI sequences, the coronal slice was positioned at approximately $3.2 \mathrm{~mm}$ posterior to bregma, using an initial FLASH localiser image. This positioning was chosen because it contained a good cross-section through the hippocampus. 


\section{TEM images of PSSS-Mag nanocomposites}
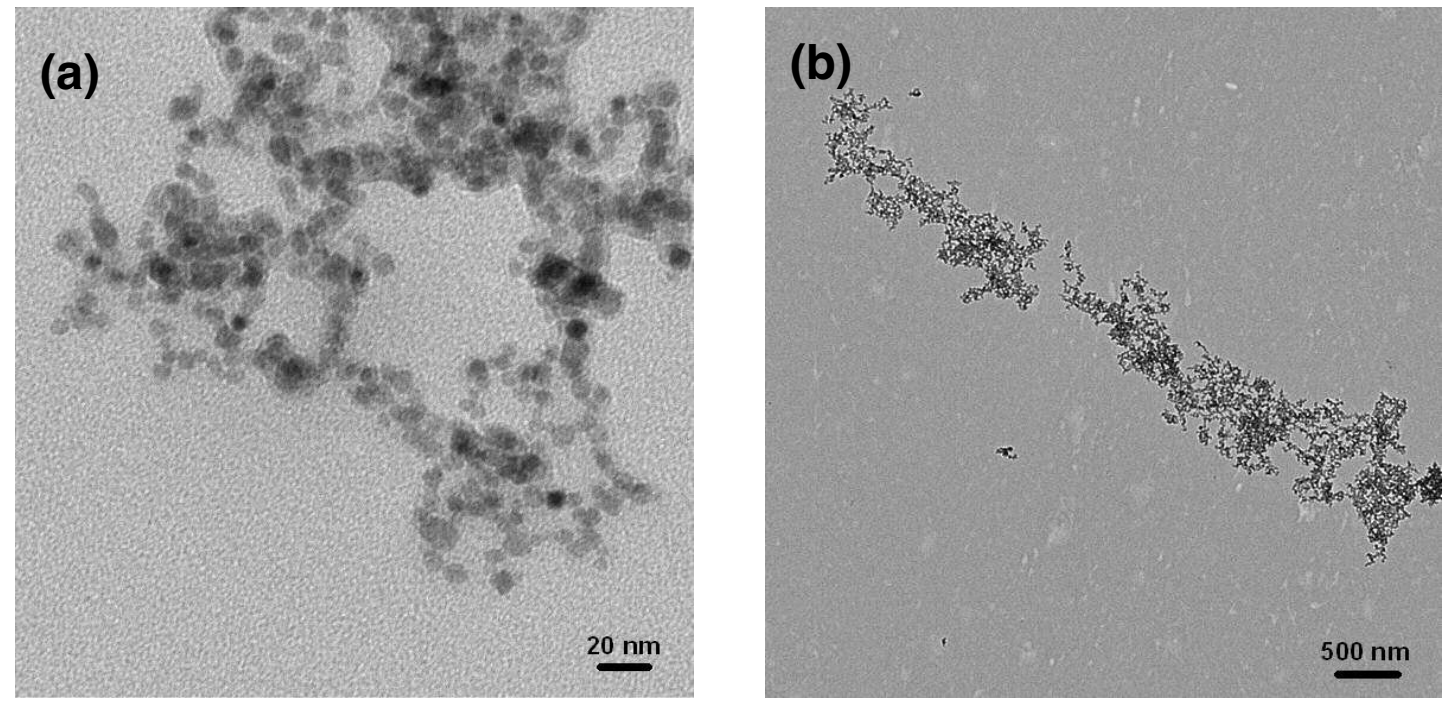

PSSS-Mag1: (a) dried without magnetic field and (b) dried in the presence of an external $0.5 \mathrm{~T}$ magnetic field.
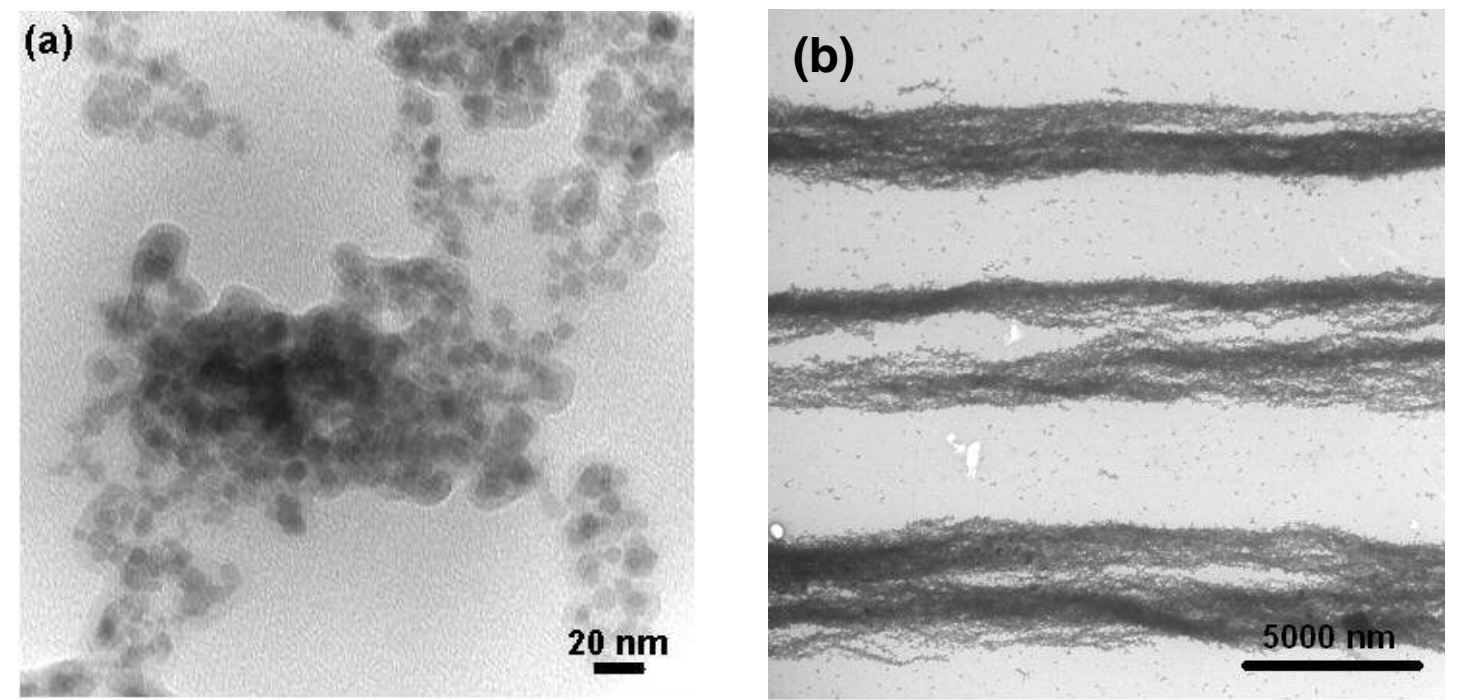

PSSS-Mag2: (a) dried without magnetic field and (b) dried in the presence of an external $0.5 \mathrm{~T}$ magnetic field. 

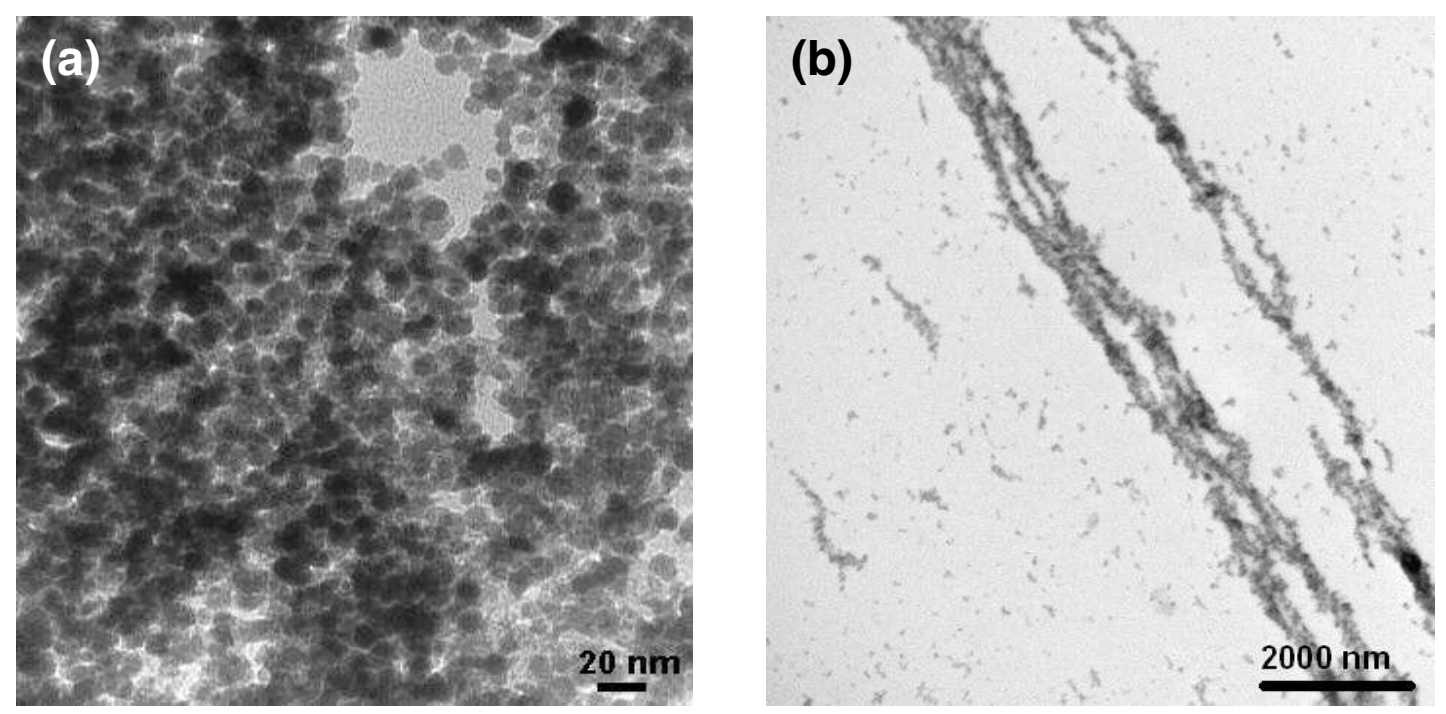

PSSS-Mag3: (a) dried without magnetic field and (b) dried in the presence of an external $0.5 \mathrm{~T}$ magnetic field 


\section{IR spectra of PSSS-Mag nanocomposites}

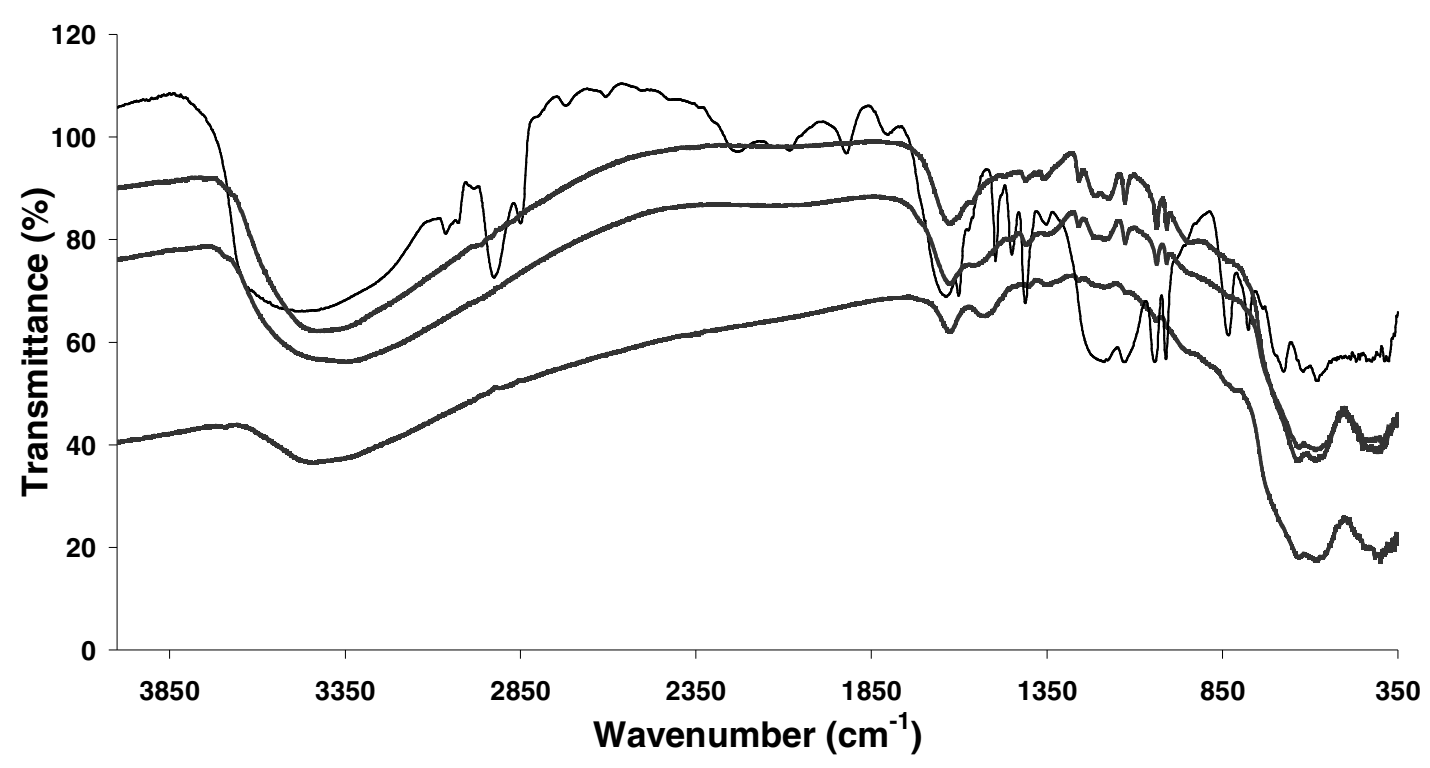

The spectrum for pure PSSS reveals stretches for C-H bonds at $2921 \mathrm{~cm}^{-1}$ and out of plane $\mathrm{C}-\mathrm{H}$ stretches at 1040 and $1009 \mathrm{~cm}^{-1}$. Stretches for the $\mathrm{CH}_{2}$ polymer backbone are found at 1495 and $1446 \mathrm{~cm}^{-1}$, while aromatic C-C stretches are seen at $1633 \mathrm{~cm}^{-1}$. The stretches for R-SO ${ }_{2}-\mathrm{OR}$ are found at 1409 and $1188 \mathrm{~cm}^{-1}$ and S-O stretches are seen at 828 and $773 \mathrm{~cm}^{-1}$. For the PSSS-Mag samples (top to bottom 1, 2 and 3), stretches for aromatic $\mathrm{C}-\mathrm{C}$ bonds, $\mathrm{CH}_{2}$ bonds, sulfonate bonds and aromatic $\mathrm{C}-\mathrm{H}$ bonds are still seen. 
4. Raman spectra of PSSS-Mag nanocomposites

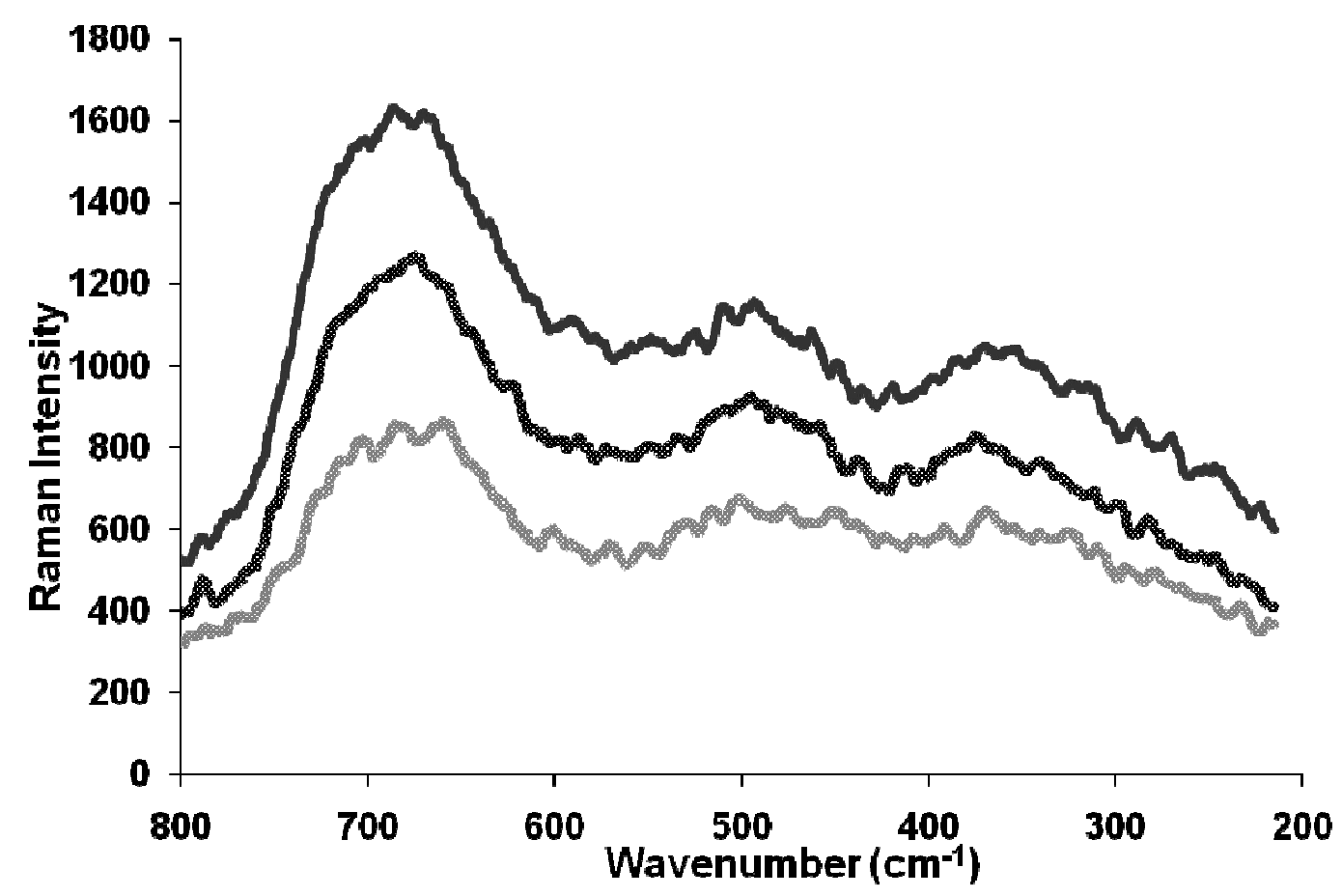

The presence of magnetite was confirmed in all samples (top to bottom, PSSS-Mag1, PSSS-Mag2 and PSSS-Mag3). Peaks found at $680 \mathrm{~cm}^{-1}$ and $496 \mathrm{~cm}^{-1}$ are in good agreement with the literature values for magnetite. ${ }^{1,2}$

1. Shebanova, O.N.; Lazor, P.; J. Raman Spec. 2003, 34, 845.

2. Shebanova, O.N.; Lazor, P.; J. Solid State Chem. 2003, 174, 424. 


\section{XRD patterns of PSSS-Mag nanocomposites}

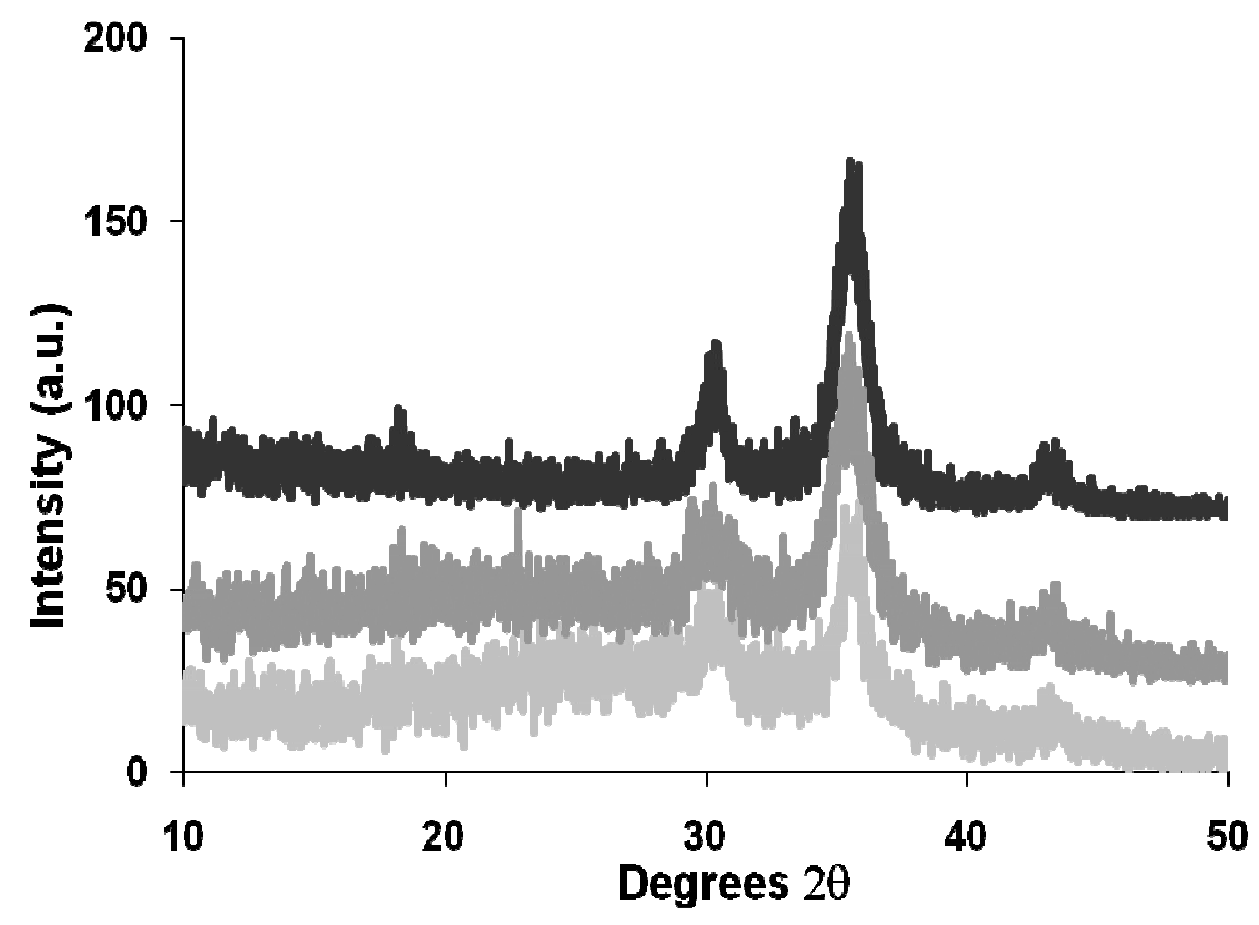

XRD patterns for PSSS-Mag samples (from top to bottom, 1, 2 and 3) showing the presence of iron oxide magnetite phase. 
6. Measurements of the spin-lattice relaxivity, $r_{1}$, in the magnetic field from $10 \mathrm{kHz}$ - $20 \mathrm{MHz}$

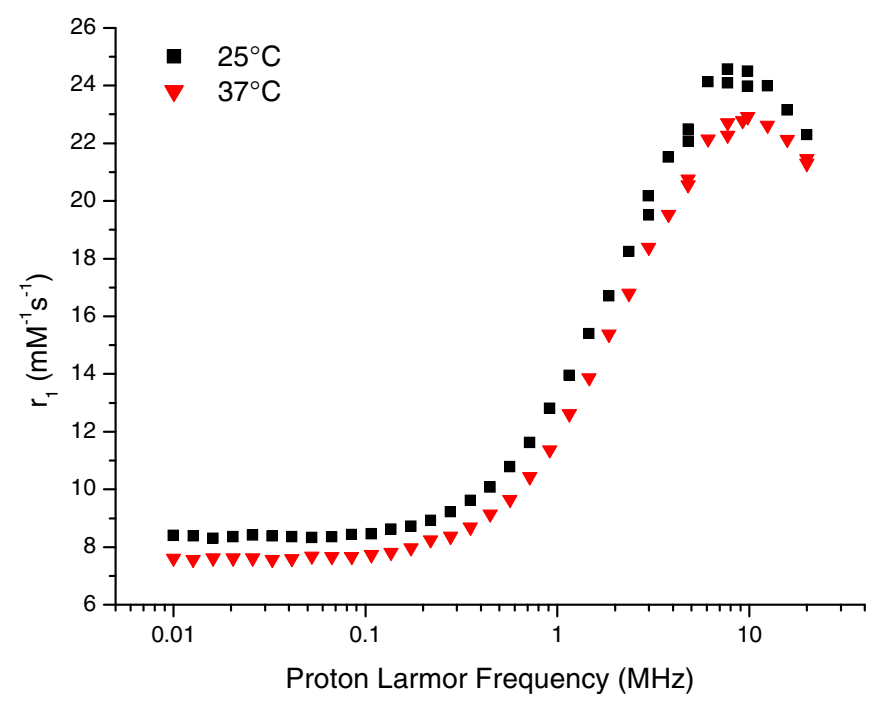

NMRD relaxation curves of PSS-Mag1 nanocomposite in water at 25 and $37^{\circ} \mathrm{C}$.

Table 2: Field dependent longitudinal and transverse relaxation data for magnetic fluid PSSS-Mag1

\begin{tabular}{|c|c|c|c|c|c|c|}
\hline $\begin{array}{c}\text { Temperature } \\
(\mathrm{K})\end{array}$ & $\begin{array}{c}\text { Field } \\
(\mathrm{MHz})\end{array}$ & $\begin{array}{c}\mathrm{T}_{1} \\
(\mathrm{~ms})\end{array}$ & $\begin{array}{c}\mathrm{T}_{2} \\
(\mathrm{~ms})\end{array}$ & $\begin{array}{c}\mathrm{r}_{1} \\
\left(\mathrm{~s}^{-1} \mathrm{mM}^{-1}\right)\end{array}$ & $\begin{array}{c}\mathrm{r}_{2} \\
\left(\mathrm{~s}^{-1} \mathrm{mM}^{-1}\right)\end{array}$ & $\begin{array}{c}\mathrm{r}_{1} / \mathrm{r}_{2} \\
\text { ratio }\end{array}$ \\
\hline 298 & 9.25 & 29.7 & 5.93 & 24.5 & 123.6 & 0.19 \\
\hline 310 & 9.25 & 23.2 & 5.55 & 22.5 & 132.4 & 0.17 \\
\hline 310 & 58.50 & 98.3 & 8.19 & 7.2 & 89.4 & 0.08 \\
\hline
\end{tabular}

The spin-spin relaxivity $r_{2}$, the specific relaxation enhancement of the samples as constituted, is significantly greater than the spin-lattice relaxivity, $\mathrm{r}_{1}$, at all fields and temperatures. We measured $r_{1}$ and $r_{2}$ values of 7.2 and $89.4 \mathrm{~s}^{-1} \mathrm{mM}^{-1}$, respectively, at $37{ }^{\circ} \mathrm{C}$ and $59 \mathrm{MHz}$ i.e. in the clinical MRI range. These observations are as expected ${ }^{3}$ given that the hydrodynamic size of the clusters is significantly greater than the superparamagnetic core size. Thus at $37{ }^{\circ} \mathrm{C}$, the $\mathrm{r}_{1} / \mathrm{r}_{2}$ ratio is 0.17 at $9 \mathrm{MHz}$, dropping to 0.08 at $59 \mathrm{MHz}$. As expected both $\mathrm{r}_{1}$ and $\mathrm{r}_{2}$ are very weakly temperature dependent in the range $25-37{ }^{\circ} \mathrm{C}$.

${ }^{3}$ Roch, A., Muller, R. N. and Gillis, P., J. Chem. Phys., 1999, 110, 5403. 


\section{MR images of rat administered with PSSS-Mag1}

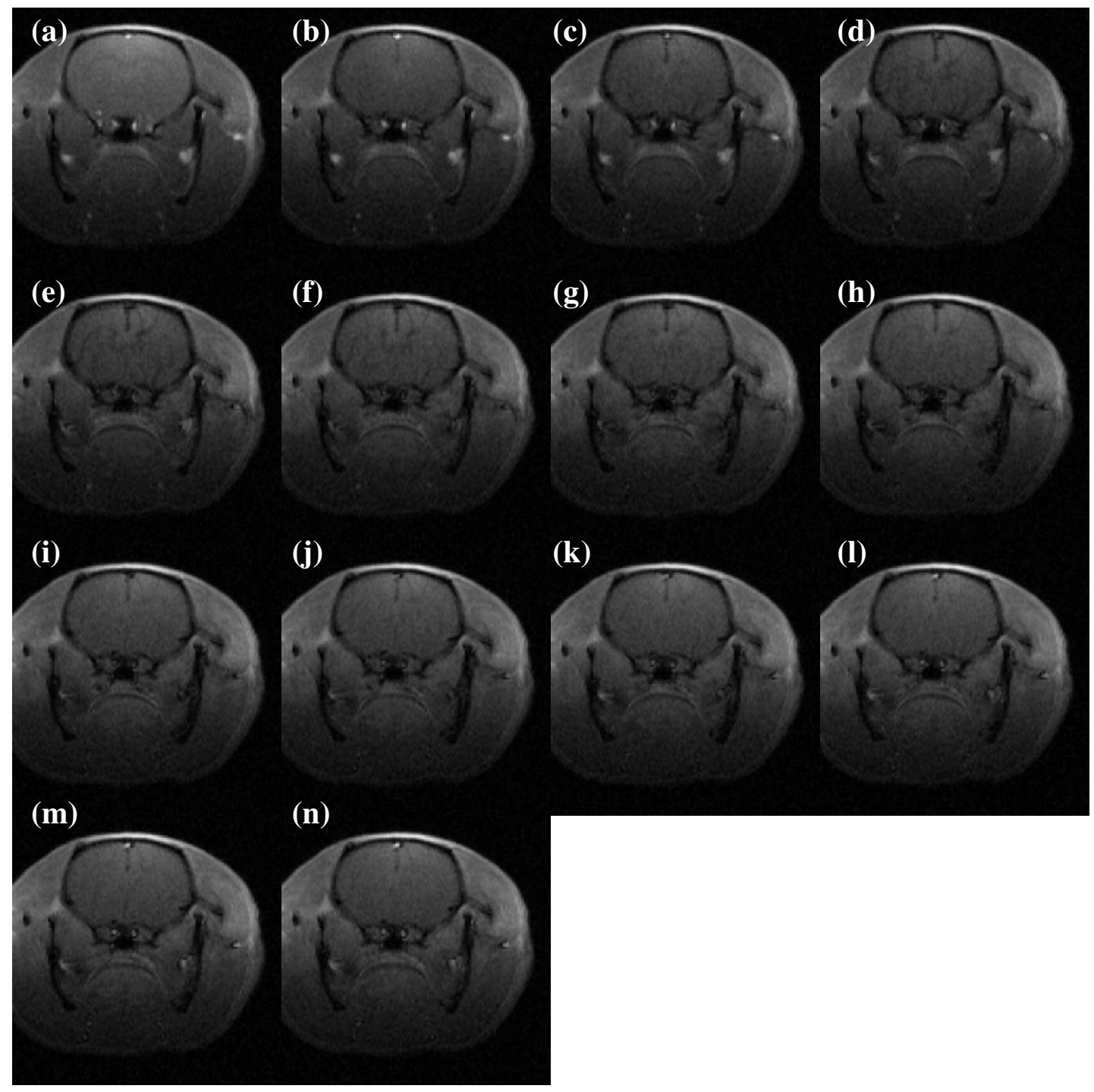

Image 7 (a) shows a baseline image before any magnetic fluid has been injected. At image 7 (b), $0.4 \mathrm{ml}$ of the particles has been injected and this shows the last baseline image where none of the bolus has yet reached the brain. At image 7(c), the bolus is entering the brain - the sinus saggitalus vein (the white dot in the centre of the brain) begins to be occluded. This is the bolus passing through the brain. Over the next few images, the S.S. vein is completed occluded with the nanoparticles and there is also a darkening of the entire brain, indicating the passage of the bolus through all the blood vessels. By image 7(1), the vein is now white again, indicating that the bolus has passed through. 
8. MR videos of rat administered with PSSS-Mag1 (see attached files)

A movie loop of the images from Figure 7. 